\section{Genetics and neuroscience of human neurobehavioral syndromes}

\author{
Genetics and Genomics of Neurobehavioral \\ Disorders \\ Gene S Fisch \\ Humana Press Inc., Totowa, New Jersey; 2003. 428 pp. \\ Price \$125.00, hardback. ISBN 1-58829-045-X
}

Heredity (2003) 91, 540-541. doi:10.1038/sj.hdy.6800367

Reviewed by WM Mc Mahon

Genetic studies of human cognition and behavior have fascinated scientists, philosophers and the public from ancient times to the present. Modern studies have approached the topic with three types of experiments: linkage studies in subjects with psychiatric illness, quantitative trait linkage studies of normal populations, definition of phenotypes that occur in individuals with learning and behavior problems due to known chromosomal deletion or duplication syndromes. Neuroscientists are hybridizing these three approaches, and using knockout mice and other organisms as models for studying human neurobehavioral disorders. This exciting progress challenges individual scientists with the breadth of methodology, uncertainty of limitations and pace of discovery. To address this challenge, Gene Fisch set out with a lofty goal as editor: 'to provide the reader with a clear and comprehensive account of how genetic abnormalities, neurobiology and neuropsychology work in concert to manifest cognitive-behavioral dysfunction'. He recruited international experts to summarize the rapidly accumulating knowledge of genetic defects associated with human mental retardation and other behavioral disorders. The 13 chapters comprising this book provide a terrific survey of this field.

The material is presented in four topical sections. Part I introduces the history, neuroanatomy, use of animal models and theoretical aspects of neurogenetics and behavioral phenotypes. Part II devotes six chapters to the review of seven neurobehavioral disorders resulting from defects on autosomes. Part III summarizes $\mathrm{X}$-linked nonsyndromal disorders, and Part IV focuses on syndromal disorders linked to the $X$ chromosome. While individual chapters serve as autonomous units of information, the overall organization of the book as a whole multiplies the value of individual chapters. Among the most interesting chapters were those in Part I on the history of the field, the relevant neuroanatomy, the use of animal models and the study of behavioral phenotypes. However, the lessons imparted in Part I may have been more apparent had the chapters utilized tables and illustrations to enliven their textual material. Parts II-IV include pictures of dysmorphic facies, schematic diagrams and radiographic images that make the disorder-specific chapters more readable. While all of the chapters are strong, those summariz ing Tuberous Sclerosis, Prader-Willi and Angelman
Syndrome, Williams-Beuren Syndrome, Fragile X syndrome and Rett Syndrome are outstanding. Following are brief synopses of chapters that caught my interest.

In the first chapter, Fisch summarizes the history of this field. The story is one of historical counterpoint, in which a theoretical theme is stated, then restated as its opposite. In the beginning, Plato espoused his theory that all knowledge was inborn, only to be opposed by his student, Aristotle, who argued that human knowledge arose from processing environmental stimuli through common sense, memory and imagination. Subsequent theories of brain function were variations on the themes of brain localization, dysmorphology, the measurement of intellectual function, the inheritance of intelligence and mental retardation. Important historical characters are mentioned as both discovering truth and supporting racism, phrenology or other false claims.

The introductory chapter on mouse models by Costa, Elgersma and Silva summarizes how molecular, physiological and behavioral studies in animal models may 'shed light on the etiology of cognitive deficits associated with genetic disorders in humans' (p. 39). The authors explain both the advantages and limitations of mouse models for integrating knowledge from molecular, neurophysiological and behavioral approaches. They emphasize that understanding of the relationship between synaptic changes and learning will require integrating results from multiple experimental approaches. Specific examples involving genes, synaptic plasticity and cognition are used to compare human diseases such as fragile $X$ mental retardation or neurofibromatosis type 1 (NF1) and homologous mouse knockouts. The role of CaMKII in long-term potentiation illustrates the criteria proposed by the authors for assigning functions to proteins. In the final chapter of the introductory section, Johnathan Flint surveys the progress and limitations in defining behavioral phenotypes in humans and extending them using animal models.

Kieran C Murphy's review of velo-cardio-facial syndrome is concise, clear and interesting. Given the estimated population prevalence (1/4000 live births), this syndrome may be the most relevant for understanding traditional psychiatric disorders. Haploinsufficiency of the 22q11 region has been associated with schizophrenia, mood disorder, attention-deficit/hyperactivity disorder and learning disabilities. The chapter includes a thoughtful critique of past methodology and needs for future studies.

Individuals with Williams-Beuren Syndrome manifest an unusual profile of learning strengths and deficits, along with cardiac, connective tissue and other clinical problems. The syndrome results from deletion of elastin and other contiguous genes on chromosome 7q11.23. Monica Bayes and Luis A Perez provide a brilliant discussion of the intricacies of contiguous gene deletion mapping against phenotypic variants. Their chapter is a model integration of facial dysmorphology, neuropsychological deficits and strengths, gene mapping and mouse knockout results for this disorder. 
Overall, this volume constitutes a well-focused snapshot of this complex and quickly developing field. Neuroscientists, behavioral geneticists, psychologists, psychiatrists and neurologists interested in the topic of genes, learning and behavior should find it informative.
WM McMahon

University of Utah, Division of Child and Adolescent Psychiatry, 421 Wakara Way, suite 143, Salt Lake City, Utah 84108, USA

E-mail: william.mcmahon@hsc.utah.edu 